\title{
Retrobulbar circulation in patients with age-related maculopathy
}

\begin{abstract}
Purpose To investigate retrobulbar circulation in stages of age-related maculopathy (ARM) and in normal fellow eyes of patients with unilateral exudative ARM.

Methods Color Doppler Imaging was used to measure circulatory parameters (peak-systolic velocity-PSV, end-diastolic velocity-EDV, pulsatility index-PI and resistivity indexRI) in the central retinal, posterior ciliary and ophthalmic arteries of 44 patients with age-related maculopathy (ARM) and 32 control subjects. Patients with ARM consisted of 11 with early ARM and 33 with late ARM. Twenty-one patients from the exudative ARM group were also included in a study of unilateral exudative ARM circulation.
\end{abstract}

${ }^{1}$ Department of Ophthalmology University of Tokyo School of Medicine Tokyo, Japan

${ }^{2}$ Department of Ophthalmology University St Cyril and Methodius

Faculty of Medicine

Skopje

Republic of Macedonia

Correspondence:

G Dimitrova

Department of

Ophthalmology

University of Tokyo

School of Medicine

7-3-1 Hongo

Bunkyo-ku

Tokyo 113-0033

Japan

Tel: 81338155411

ext 3499

Fax: 81338170798

E-mail: galina-tky@

umin.ac.jp
Results In the study of patients with ARM divided into stages, the PI and RI in the posterior ciliary artery of patients with late ARM were significantly higher than that of the control group $(P=0.0064 ; P=0.0124)$. In the early ARM group, circulatory parameters did not differ significantly from those of the control group.

In the study of unilateral exudative ARM, the affected eye showed significantly higher RI, as compared to the control group ( $P=$ 0.0157), and the fellow eye had significantly decreased EDV as compared to the control group $(P=0.0164)$. There was no significant difference in circulatory parameters between the affected and normal fellow eyes of patients with unilateral exudative ARM. Conclusion PI and RI in patients with late ARM and EDV in fellow eyes of patients with unilateral exudative ARM showed significant changes in the posterior ciliary artery compared to normal controls. Altered circulation in the posterior ciliary artery may be involved in the pathogenesis of ARM.
G Dimitrova ${ }^{1,2}$, Y Tamaki ${ }^{1}$ and $\mathrm{S}$ Kato $^{1}$

Eye (2002) 16, 580-586. doi:10.1038/

sj.eye. 6700161

Keywords: age-related maculopathy; ultrasound; angiogenesis; posterior ciliary artery; central retinal artery; ophthalmic artery

\section{Introduction}

Age-related maculopathy (ARM) is a major cause of blindness in developed countries. Morphologic alterations in the retinal pigment epithelium (RPE)-Bruch's membrane complex and the choroid have been described. ${ }^{1-4}$ Although the etiology of ARM is not clearly understood, vascular irregularities are present in ARM and a blood circulatory dysfunction has been proposed in the pathogenesis of this disease. ${ }^{5-9}$

Because a number of reports ${ }^{10-13}$ have provided qualitative data suggesting abnormalities of the choroidal circulation in ARM, quantitative assessment of the choroidal circulation has been thought to be important for investigating the etiologic role of ocular perfusion in the development of ARM.

Two studies using color Doppler imaging (CDI) demonstrated an increased pulsatility index (PI) in the central retinal artery, posterior ciliary artery and ophthalmic artery in a group of exudative and non-exudative ARM patients, ${ }^{6}$ and decreased end-diastolic velocity (EDV) in the posterior ciliary artery in non-exudative ARM patients. ${ }^{7}$ A pulsatile ocular blood flow study measured patients with non-exudative ARM and exudative ARM in separate groups and found decreased choroidal flow only in exudative ARM. ${ }^{14}$ However, a study using CDI, that investigates the circulation not only in the posterior ciliary artery that supplies the choroid, but also in the central retinal and ophthalmic artery of patients with early and late ARM divided in separate groups has not been done as yet. 
On the other hand, unilateral exudative ARM reportedly has an incidence of progression to exudative ARM in the fellow eye of $3-28 \%$ in 1 year ${ }^{13}$ and $42 \%{ }^{15}$ in 5 years. In a fluorescein angiographic study, choroidal perfusion defects were detected in $42 \%$ of fellow eyes in unilateral exudative ARM patients and electroretinographic (ERG) abnormalities were also present in those eyes. ${ }^{11}$ Indocyanine-green studies reported prolonged choroidal perfusion in $11 \%{ }^{13}$ and hyperfluorescent areas in $33 \%$ of fellow eyes in unilateral exudative ARM. ${ }^{12}$ However, the circulation in the fellow eye in unilateral ARM has not been investigated with quantitative methods.

This study was designed to investigate retrobulbar circulation in the early and late stages of ARM and in normal fellow eyes of patients with unilateral exudative ARM.

\section{Subjects and methods}

The study was performed in accordance with the standards of the ethics committee of the University of Tokyo School of Medicine. All subjects were informed about the nature of the study and gave their consent to be included. The research followed the tenets of the Declaration of Helsinki.

Forty-four patients with ARM and 32 age-matched control subjects were enrolled in the study. The subjects were randomly chosen from among patients who came for regular check-ups in the macular outpatient clinic at the Department of Ophthalmology, Tokyo University Hospital. Thirteen hypertensive patients with ARM and six control subjects had systemic hypertension. However, their hypertension was controlled and there were no significant differences in blood pressure among the groups. Patients with other diseases of the eye possibly affecting the retrobulbar circulation, such as diabetic retinopathy and glaucoma, and those with a history of laser treatment or intraocular surgery were excluded. We selected age-matched control subjects from among patients who had cataracts but no other ocular diseases or patients with one healthy eye and only minor ocular diseases, such as conjuctivitis and keratitis, in the other eye. In the latter case, the healthy eye was examined for this study. The patients underwent assessment of best-corrected visual acuity after standardized refraction. Intraocular pressure (IOP) was measured by Goldman tonometry. Brachial artery systolic (BPs) and diastolic (BPd) blood pressures were determined by an automatic blood pressure apparatus. The mean brachial artery pressure $(\mathrm{BPm})$ was calculated as follows $\mathrm{BPm}=\mathrm{BPd}+1 / 3(\mathrm{BPs}-\mathrm{BPd})$

\section{(1) Study of retrobulbar circulation in stages of} ARM

The patient group was subdivided as follows: 11 patients with early ARM (drusen $\geq 63 \mu \mathrm{m}$ and/or atrophy of the RPE $<175 \mu \mathrm{m}$ and/or proliferation of the RPE), and 33 patients with late ARM. Patients with late ARM consisted of seven patients with pigment epithelium detachment, 18 patients with choroidal neovascularization and eight patients with disciform scar. The patients having choroidal neovascularization consisted of four patients with predominantly classic and 14 patients with predominantly occult form of choroidal neovascularization. Patients with geographical atrophy were excluded from the study. The group of ARM patients included 8 women and 36 men, while the control group included 17 women and 15 men. Classification of the different stages of ARM was based on ophthalmoscopic examinations, including stereoscopic biomicroscopy and fluorescein and/or indocyanine green angiography, according to the international classification and grading system of the international ARM epidemiological study group. ${ }^{16}$ The eye that had a worse visual acuity was chosen for the analysis of patients with various stages of ARM. The early ARM group included patients who had either one eye with early ARM and a healthy fellow eye, or in whom both eyes had early ARM.

\section{(2) Study of retrobulbar circulation in patients with unilateral exudative ARM}

In the group of patients with late ARM, 21 who had unilateral exudative ARM and a fellow eye without ARM were included in the study of unilateral exudative ARM circulation. Control subjects $(n=32)$ were the same as above.

\section{Measurements of ocular blood flow}

All the measurements were performed with a CDI set: Powervision SSA-380 A (Toshiba, Tokyo, Japan) using a $7 \mathrm{MHz}$ transducer. One observer, masked to the subject's status, did all the measurements (GD).

The patients were examined while in a sitting position, as described elsewhere. ${ }^{17}$ Patients fixed their gazes about $15 \mathrm{~cm}$ in front of their faces and the transducer coupled with sterile ophthalmic gel was applied to the upper lids. We measured the following retrobulbar blood vessels: central retinal artery, posterior ciliary artery and ophthalmic artery. The central retinal artery appeared $10-15 \mathrm{~mm}$ away from the optic disc and was visible inside the optic nerve, until its entrance into the globe. Because the blood 
flow velocity in the central retinal artery over the lamina cribrosa is usually higher than normal, the receiver's gate was placed about $5 \mathrm{~mm}$ away from this structure.

The posterior ciliary artery was found parallel to the optic nerve at about the same distance from the fundus as the central retinal artery and vein. The ophthalmic artery was usually found nasal to the optic nerve.

The hemodynamic parameters measured in the aforementioned blood vessels were peak systolic blood velocity (PSV) and EDV. The PI and resistivity index (RI) were calculated as follows:

$\mathrm{PI}=(\mathrm{PSV}-\mathrm{EDV}) / \mathrm{MV}$

where MV is mean velocity.

$\mathrm{RI}=(\mathrm{PSV}-\mathrm{EDV}) / \mathrm{PSV}$

The CDI method allows for accurate PI and RI measurement because its values do not depend on the Doppler angle. We attempted to maintain the Doppler angle as parallel as possible to the measured blood vessel in order to obtain more accurate blood velocity data.

\section{Statistical analysis}

ANOVA and the unpaired $t$-test were used for the analysis of differences in patients' systemic characteristics. The paired $t$-test was used for the analysis of circulatory differences between the normal fellow and the exudative eye in unilateral ARM. The non-parametric Kruskal-Wallis test was used for the analysis of circulatory parameters in patients with different stages of ARM and for comparison of circulatory characteristics between both eyes of patients with unilateral exudative ARM and those of the control group. Dunn correction was applied to test the significance of differences between the groups in both analyses. $P$ values less than 0.0167 were taken as statistically significant.

\section{Results}

\section{(1) Study of retrobulbar circulation in the early and late stage of ARM (Table 1)}

There were no significant differences in systemic characteristics (age, BPm, pulse) and IOP among ARM patient groups and controls. (Table 2). In five control subjects and 14 patients with ARM, we could not obtain the velocity wave of the ophthalmic artery and in one control subject we could not obtain the velocity wave of the posterior ciliary artery.

In the posterior ciliary artery, the PI and RI were significantly higher in the late ARM group than in controls $(P=0.0064 ; P=0.0124)$. In the central retinal artery and in the ophthalmic artery, no parameter differed significantly among the groups.

\section{(2) Study of retrobulbar circulation in patients with unilateral exudative ARM (Table 3)}

Systemic characteristics did not differ significantly between unilateral exudative ARM patients and control subjects. Central retinal artery and posterior ciliary artery velocities were measurable in both eyes in all subjects, while the ophthalmic artery velocity wave could not be obtained in three fellow eyes.

In the posterior ciliary artery, the EDV was significantly lower in the posterior ciliary artery of the fellow eye $(P=0.0164)$ and was also lower in the exudative eye ( $P=0.0668$ ) compared to that of the control group. The RI in the posterior ciliary artery was significantly higher $(P=0.0157)$ in the exudative eyes and was also higher $(P=0.0537)$ in the fellow eyes of unilateral exudative ARM than in the control. The PI in the posterior ciliary artery in eyes with exudative ARM tended to be higher in both the exudative eye $(P=0.0191)$ and the fellow eye $(P=$ $0.0327)$ than in the control group.

In the central retinal and ophthalmic arteries, the circulatory parameters of patients with unilateral exudative ARM did not differ significantly from those of the control group.

There were no significant differences in any parameter between the exudative and normal fellow eyes.

\section{Discussion}

\section{(1) Study of retrobulbar circulation in stages of ARM}

Choroidal circulatory dysfunction has been found in patients with ARM that may be related to the pathology of this disease. ${ }^{5-9,14}$ In the first part of this study we demonstrated that patients with late ARM have significantly higher PI and RI in the posterior ciliary artery that perfuses the choroid than control subjects. A previous study using CDI investigated patients with ARM that consisted of early and late ARM included in one group. ${ }^{6}$ They also reported significantly increased PI in the posterior ciliary artery and in the central retinal artery. Ciulla et $a l^{7}$ also using CDI demonstrated significantly decreased EDV in the central retinal and posterior ciliary arteries in patients with non-exudative ARM, including patients with early ARM (some of which were fellow eyes of unilateral 
Table 1 Circulatory parameters in control subjects and ARM patients divided in stages

\begin{tabular}{|c|c|c|c|c|}
\hline & & Control & Early ARM & Late ARM \\
\hline PSV & CRA & $8.48 \pm 2.36(32)$ & $7.08 \pm 1.81(11)$ & $8.78 \pm 2.80$ \\
\hline \multirow[t]{2}{*}{$(\mathrm{cm} / \mathrm{s})$} & PCA & $8.23 \pm 2.83$ & $7.83 \pm 2.34(11)$ & $7.91 \pm 2.00$ \\
\hline & $\mathrm{OA}$ & $22.26 \pm 5.58$ & $23.48 \pm 5.90(9)$ & $23.26 \pm 6.76(21)$ \\
\hline EDV & CRA & $2.59 \pm 0.84$ & $2.34 \pm 0.67(11)$ & $2.47 \pm 0.64$ \\
\hline \multirow[t]{2}{*}{$(\mathrm{cm} / \mathrm{s})$} & PCA & $3.11 \pm 1.02(31)$ & $2.99 \pm 1.06(11)$ & $2.62 \pm 0.71$ \\
\hline & $\mathrm{OA}$ & $3.89 \pm 1.54(27)$ & $4.54 \pm 2.00(9)$ & $4.11 \pm 2.30(21)$ \\
\hline \multirow[t]{3}{*}{ PI } & CRA & $1.22 \pm 0.28$ & $1.18 \pm 0.19$ & $1.30 \pm 0.24$ \\
\hline & PCA & $0.97 \pm 0.17(31)$ & $0.97 \pm 0.23^{*}(11)$ & $1.13 \pm 0.26$ \\
\hline & $\mathrm{OA}$ & $1.90 \pm 0.57(27)$ & $1.72 \pm 0.36$ & $0.66 \pm 0.08$ \\
\hline \multirow[t]{3}{*}{ RI } & CRA & $0.69 \pm 0.08$ & $0.67 \pm 0.05$ & $0.71 \pm 0.06$ \\
\hline & PCA & $0.61 \pm 0.06(31)$ & $0.61 \pm 0.08^{*}(11)$ & $0.66 \pm 0.08$ \\
\hline & OA & $0.82 \pm 0.06(27)$ & $0.81 \pm 0.07$ & $0.83 \pm 0.06(21)$ \\
\hline
\end{tabular}

Mean \pm SD

PSV, peak systolic velocity; EDV, end-diastolic velocity; PI, pulsatility index; RI, resistivity index; CRA, central retinal artery; PCA, posterior ciliary artery; OA, ophthalmic artery.

*Significant difference vs control group, $P<0.0167$ (Kruskall-Wallis test, statistical significance with Dunn correction).

Table 2 Patients' and control subjects' clinical characteristics

\begin{tabular}{lccc}
\hline Number $(n)$ & $\begin{array}{c}\text { Control } \\
(32)\end{array}$ & $\begin{array}{c}\text { Early ARM } \\
(11)\end{array}$ & $\begin{array}{c}\text { Late ARM } \\
(33)\end{array}$ \\
\hline $\begin{array}{l}\text { Age } \\
\text { (years) }\end{array}$ & $64.7 \pm 9.1$ & $65.6 \pm 7.3$ & $69.9 \pm 9.9$ \\
$\begin{array}{l}\text { Mean BP } \\
\text { (mm Hg) }\end{array}$ & $91.1 \pm 10.8$ & $95.4 \pm 12.1$ & $95.2 \pm 10.1$ \\
$\begin{array}{l}\text { Pulse rate } \\
\text { (beats/min) }\end{array}$ & $79 \pm 13$ & $79 \pm 15$ & $74 \pm 12$ \\
$\begin{array}{l}\text { IOP } \\
\text { (mmHg) }\end{array}$ & $14.05 \pm 2.4$ & $14.2 \pm 3.4$ & $14.0 \pm 2.8$ \\
\hline
\end{tabular}

Mean \pm SD.

Mean BP, mean blood pressure; IOP, intraocular pressure.
ARM), and patients with geographic atrophy. Because perfusion defects and other circulatory disturbances have been reported in previous studies in fellow eyes of unilateral ARM, ${ }^{11-13}$ we included patients in the early ARM group in our study if their fellow eye was either normal or had early ARM. The differences in the inclusion criteria impose difficulties in making a valid comparison between the present and the above mentioned studies.

Hypertension is known to be prevalent among ARM patients and blood pressure and posture have been found to affect central retinal circulation. ${ }^{18,19}$ However, blood pressure and posture during measurement have not been reported in the above mentioned studies.

Except for the inclusion criteria, we suspect these to be

Table 3 Circulatory parameters in both eyes of unilateral exudative ARM and control subjects

\begin{tabular}{|c|c|c|c|c|}
\hline & & Control & Exudative & Fellow \\
\hline PSV & CRA & $8.48 \pm 2.36(32)$ & $9.61 \pm 2.88(21)$ & $9.22 \pm 2.91(21)$ \\
\hline \multirow[t]{2}{*}{$(\mathrm{cm} / \mathrm{s})$} & PCA & $8.23 \pm 2.83$ & $8.06 \pm 1.89$ & $7.65 \pm 2.42$ \\
\hline & $\mathrm{OA}$ & $22.26 \pm 5.58$ & $23.12 \pm 6.82(10)$ & $26.59 \pm 6.73(10)$ \\
\hline EDV & CRA & $2.59 \pm 3.11$ & $2.64 \pm 0.68$ & $2.63 \pm 0.70$ \\
\hline \multirow[t]{2}{*}{$(\mathrm{cm} / \mathrm{s})$} & PCA & $3.11 \pm 1.02(31)$ & $2.64 \pm 0.74(21)$ & $2.48 \pm 0.90^{*}(21)$ \\
\hline & OA & $3.89 \pm 1.54(27)$ & $3.91 \pm 2.39(10)$ & $4.59 \pm 2.60(10)$ \\
\hline \multirow[t]{3}{*}{ PI } & CRA & $1.22 \pm 0.28$ & $1.32 \pm 0.26(21)$ & $1.25 \pm 0.25(21)$ \\
\hline & PCA & $0.97 \pm 0.17(31)$ & $1.15 \pm 0.28^{+}(21)$ & $1.14 \pm 0.34^{\ddagger}(21)$ \\
\hline & OA & $1.90 \pm 0.57(27)$ & $2.04 \pm 0.59(10)$ & $1.96 \pm 0.61(10)$ \\
\hline \multirow[t]{3}{*}{ RI } & CRA & $0.69 \pm 0.08$ & $0.72 \pm 0.06$ & $0.70 \pm 0.08$ \\
\hline & PCA & $0.61 \pm 0.06(31)$ & $0.67 \pm 0.08^{\S}(21)$ & $0.66 \pm 0.09(21)$ \\
\hline & $\mathrm{OA}$ & $0.82 \pm 0.06$ & $0.84 \pm 0.06(10)$ & $0.83 \pm 0.07(10)$ \\
\hline
\end{tabular}

Mean \pm SD.

PSV, peak systolic velocity; EDV, end-diastolic velocity; PI, pulsatility index; RI, resistivity index; CRA, central retinal artery; PCA, posterior ciliary artery; OA, ophthalmic artery.

*Significant difference $v$ s control group, $P=0.0164$

'Statistical tendency os control group, $P=0.019$.

‡Statistical tendency $v$ s control group, $P=0.0327$.

§Significant difference $v$ s control group, $P=0.0157$ (Kruskall-Wallis test, statistical significance tested with Dunn correction). 
among the possible reasons why the results from the present and the mentioned studies differ with respect to the results of the central retinal artery circulation.

A study using laser Doppler flowmetry found no significant differences in choroidal blood velocity in the center of the fovea, but did demonstrate significantly decreased foveolar choroidal blood flow in patients with early ARM. ${ }^{8}$ This study also included five early ARM eyes of patients who had unilateral exudative ARM. Similarly, differences in blood velocity between our early ARM patients and the control group were not statistically significant. However, the PI and RI in our early ARM patients did not differ from those of the control group either, suggesting absence of circulatory alteration in the posterior ciliary artery of patients with early ARM. There may be two reasons for the apparent discrepancy between our study and the above mentioned laser Doppler flowmetry study. First, the laser Doppler flowmetry measurements in the fovea correspond primarily to determinations of choriocapillary flow, ${ }^{8}$ which can be influenced by local autonomic and autoregulatory effects ${ }^{14,20,21}$ and may not correspond to the blood flow in the posterior ciliary artery. The other reason is that, as mentioned earlier, fellow eyes of unilateral exudative ARM were included. In our study, we demonstrated that choroidal circulation in fellow eyes of unilateral exudative ARM is affected and thus it may not be representative of the circulation in early ARM.

Mori et al ${ }^{14}$ investigated choroidal blood flow by the pulsatile blood flow method in patients with nonexudative and exudative ARM. The authors found no significant changes in choroidal blood flow in patients with non-exudative ARM, while choroidal blood flow was decreased in patients with exudative ARM. These data are consistent with our data showing increased resistance to choroidal blood inflow in late ARM and no significant differences between patients with early ARM and control subjects. However, as to the nonexudative group in the study of Mori et al, ${ }^{14}$ there was no information provided about the types of nonexudative ARM eyes included (early ARM, geographic atrophy or fellow eyes of unilateral exudative ARM).

To our knowledge, there have been no studies investigating retrobulbar circulation in patients divided in early and late ARM stages, and using CDI. We herein report that circulatory parameters do not differ significantly between early ARM eyes and controls, while eyes with late ARM have significantly higher PI and RI in the posterior ciliary artery than controls. These data suggest that retrobulbar circulation is not altered in early ARM. The increase of PI and RI in the posterior ciliary artery (which supplies blood to the choroid) of the exudative ARM group suggests altered choroidal circulation in this stage of ARM. However, from this study alone, we can not speculate as to whether the altered circulation is a factor or a consequence of the occurrence of exudative ARM.

Both increased ${ }^{1}$ and decreased ${ }^{22}$ choriocapillaris density and diameters have been documented in histopathologic studies of patients with ARM. Distended and tortuous choroidal veins ${ }^{1,5}$ have also been reported. The origin of the latter has been linked to the increased scleral rigidity in patients with ARM as described by Friedman et al. ${ }^{23}$ The same authors further proposed that the increased scleral rigidity might increase resistivity to choroidal blood inflow and outflow. ${ }^{7}$ Therefore, a decrease in EDV with a consequent increase of the PI and RI (see Equations 1 and 2 ) in the posterior ciliary artery could be expected with disturbed choroidal inflow.

\section{(2) Study of retrobulbar circulation in patients with unilateral exudative ARM}

High incidences of progression to exudative form in fellow eyes of unilateral exudative ARM have been reported (3-28\% in 1 year, ${ }^{13} 42 \%{ }^{15}$ and $33 \%^{12}$ in 5 years). Choroidal perfusion defects in fellow eyes have been demonstrated using indocyanine-green angiography ${ }^{12,13}$ and fluorescein angiography. ${ }^{11}$ ERGs of fellow eyes also had slower implicit times than in control age-matched subjects. ${ }^{11}$ Furthermore, histological studies have found considerable symmetry of drusen characteristics between the two eyes of patients with unilateral exudative ARM. ${ }^{24}$

In the present study, patients with unilateral exudative ARM showed significantly increased RI in the posterior ciliary artery, in the exudative eyes, as compared to control subjects. In normal fellow eyes, EDV was significantly decreased in the posterior ciliary artery, while both eyes tended to have an increased PI $(P=0.019 ; P=0.033)$ in the same artery as compared to controls. Decreased EDV and increased RI and PI are considered to represent an increase of peripheral vascular resistance. ${ }^{25}$ Furthermore, there was no significant difference in any of the circulatory parameters between normal fellow and exudative ARM eyes.

In study 1 we did not detect significant differences in the retrobulbar circulation of patients with early ARM compared to control subjects. This is consistent with the fact that the annual incidence of progression to late ARM in patients with early ARM is relatively low (1-4\%). ${ }^{26}$ On the other hand, in study 2 we detected significantly altered retrobulbar circulation in the normal fellow eyes of patients with unilateral exudative ARM, which supports previous findings of 
prolonged choroidal perfusion and ERG defects, as well as the high indices of progression to exudative ARM in fellow eyes of unilateral exudative ARM. ${ }^{11-13}$ This suggests that despite the apparent normal fundus, choroidal circulation in the fellow eye is already compromised. Therefore, normal fellow eyes in patients with unilateral exudative ARM could be treated as a subgroup with an increased risk of progression to exudative ARM.

Ciulla et al, ${ }^{27}$ in their review of the role of ocular perfusion in the pathophysiology of ARM, stated that with the available studies the causative role of the circulatory factor in ARM could not be determined. Further prospective studies are needed to confirm our findings. However, the results of the present study suggest for the first time that altered choroidal circulation may be a pathogenic factor in the progression to exudative ARM.

Progression to exudative ARM in the fellow eye in unilateral exudative ARM has disastrous effects on a patient's quality of life. The results of this and other studies may suggest that strict control of hypertension and therapy to improve choroidal blood perfusion would prove beneficial in halting the advancement of ARM.

In conclusion, we have demonstrated an increased PI and RI in the posterior ciliary artery of patients with late ARM, which suggests decreased choroidal perfusion. The increased PI and RI in the posterior ciliary artery of both fellow and affected eyes and the lack of significant differences between the two eyes of patients with unilateral ARM suggest that the normal fellow eye has deficient choroidal blood perfusion. The results from both parts of this study suggest that deficient choroidal perfusion may be a factor in progression to exudative ARM.

\section{Acknowledgements}

This study has been presented at the 105th annual meeting of the Japanese Ophthalmological Society held in Yokohama (Japan) 19th-21st April 2001. It has been funded by the Japanese Ministry of Education (Monbusho).

\section{References}

1 Spraul GW, Lang GE, Grossniklaus HE. Morphometric analysis of the choroid, Bruch's membrane, and retinal pigment epithelium in eyes with age-related macular degeneration. Invest Ophthalmol Vis Sci 1996; 37: 27242735.

2 Kornzweig AL, Eliasoph I, Feldstein M. The retinal vasculature in macular degeneration. Arch Ophthalmol 1966; 75: 326-333.
3 Green WR, Enger C. Age-related macular degeneration histopathologic studies. Ophthalmology 1993; 100: 15191535.

4 van der Schaft TL, Mooy CM, de Brujin WC, Oron FG, Mulder PGH, de Jong PTVM. Histologic features of the early stages of age-related maculopathy. Ophthalmology 1992; 99: 278-286.

5 Friedman E, Smith TR, Kuwabara T. Senile choroidal vascular patterns and drusen. Arch Ophthalmol 1963; 69: 220-230.

6 Friedman E, Krupsky S, Lane AM et al. Ocular blood flow velocity in age-related macular degeneration. Ophthalmology 1995; 102: 640-646.

7 Ciulla TA, Harris A, Chung HS et al. Color Doppler imaging discloses reduced ocular blood flow velocities in nonexudative age-related macular degeneration. Am J Ophthalmol 1999; 128: 75-80.

8 Grunwald JE, Hariprasad SM, DuPont J et al. Foveolar choroidal blood flow in ARM. Invest Ophthalmol Vis Sci 1998; 39: 385-390.

9 Remsch H, Spraul CW, Lang GK, Lang GE. Changes of retinal capillary blood flow in age-related maculopathy. Graefe's Arch Clin Exp Ophthalmol 2000; 238: 960-964.

10 Pauleikoff D, Chen JC, Chisholm IH, Bird AC. Choroidal perfusion abnormality with age-related Bruch's membrane change. Am J Ophthalmol 1990; 109: 211-217.

11 Remulla JFC, Gaudio AR, Miller S, Sandberg MA. Foveal electroretinograms and choroidal perfusion characteristics in fellow eyes of patients with unilateral neovascular agerelated macular degeneration. $\mathrm{Br}$ J Ophthalmol 1995; 79: 558-561.

12 Haruyama M, Yuzawa M. Hyperfluorescent areas observed in late-phase indocyanine green angiography (IA) of IA findings in fellow eyes of unilateral age-related macular degeneration. J Jpn Ophthalmol Soc 2000; 104: 805-812.

13 Hanutsaha P, Guyer DR, Yanuzzi LA et al. Indocyaninegreen videoangiography of drusen as a possible predictive indicator of exudative maculopathy. Ophthalmology 1998; 105: 1632-1636.

14 Mori F, Konno S, Hikichi T, Yamaguchi Y, Ishiko S, Yoshida A. Pulsatile ocular blood flow study: decreases in exudative age related macular degeneration. $\mathrm{Br} \mathrm{J}$ Ophthalmol 2001; 85: 531-533.

15 Macular photocoagulation study group. Risk factors for choroidal neovascularization in the second eye of patients with juxtafoveal or subfoveal choroidal neovascularization secondary to age-related macular degeneration. Arch Ophthalmol 1997; 115: 741-747.

16 The international ARM epidemiological study group. An international classification and grading system for agerelated maculopathy and age-related macular degeneration. Surv Ophthalmol 1995; 39: 367-374.

17 Hasegawa S, Nagahara M, Araie M. Color Doppler imaging in sitting patients. The 52nd Japanese Clinical Ophthalmology Congress 1998. Congress program page 259.

18 Rassam SMB, Patel V, Kohner EM. The effect of experimental hypertension on retinal vascular autoregulation in humans: a mechanism for the progression of diabetic retinopathy. Exp Physiol 1995; 80 53-68.

19 Evans DW, Harris A, Garrett M, Chung HS, Kagemann L. Glaucoma patients demonstrate faulty autoregulation 
of ocular blood flow during posture change. $\mathrm{Br}$ Ophthalmol 1999; 83: 809-813.

20 Chou PI, Lu DW, Chen JT. Bilateral superior cervical ganglionectomy increases choroidal blood flow in the rabbit. Ophthalmologica 2000; 214: 421-425.

21 Roff EJ, Harris A, Chung HS et al. Comprehensive assessment of retinal choroidal and retrobulbar haemodynamics during blood gas perturbation. Graefe's Arch Clin Exp Ophthalmol 1999; 237: 984-990.

22 Ramrattan RS, van der Schaft TL, Mooy CM, de Brujin WC, Mulder PGH, de Jong PTVM. Morphometric analysis of Bruch's membrane, the choriocapillaris and the choroid in aging. Invest Ophthalmol Vis Sci 1994; 35: 2857-2864.

23 Friedman E, Ivry M, Ebert E, Glynn R, Gragoudas E,
Seddon J. Increased scleral rigidity and age-related macular degeneration. Ophthalmology 1988; 96: 104-108.

24 Pauliekhoff D, Barondes MJ, Minassian D, Chisholm IH, Bird AC. Drusen as risk factors in age-related macular disease. Am J Ophthalmol 1990; 109: 38-43.

25 Evans W, Harris A, Danis RP, Arend O, Martin B. Altered retrobulbar vascular reactivity in early diabetic retinopathy. Br J Ophthalmol 1997; 81: 279-282.

26 Berger JW, Fine SL, Maguire MG. Age-Related Macular Degeneration. Mosby: St Louis, 1999, p 24.

27 Cuilla TC, Harris A, Martin BJ. Ocular perfusion and agerelated macular degeneration. Acta Ophthalmol Scand 2001; 79: $108-115$. 